\title{
PERIODIC SOLUTIONS AND EQUILIBRIUM STATES FOR FUNCTIONAL DIFFERENTIAL INCLUSIONS WITH NONCONVEX RIGHT-HAND SIDE
}

\author{
$\mathrm{BY}$ \\ LI YONG, ZHOU QINDE, AND LU XIANRUI \\ Department of Mathematics, Jilin University, Changchun 130023, P. R. China
}

\begin{abstract}
In this paper, we present a general existence theorem for periodic solutions for functional differential inclusions with nonconvex right-hand side. As an application, we prove that for a multivalued system with nonconvex right-hand side, permanence implies the existence of a pre-equilibrium state, which answers an open problem proposed by Hutson.
\end{abstract}

1. Introduction. Deterministic modelling in the biological sciences often leads to ordinary differential equations, for example, ecological differential equations with state space $R_{+}^{n}$ :

$$
x_{i}^{\prime}=x_{i} f_{i}(x), \quad i=1, \ldots, n, x=\left(x_{1}, \ldots, x_{n}\right) .
$$

For such systems, it is quite important to discover whether permanence implies the existence of equilibrium states or not. From a biological point of view, it is reasonable to expect an affirmative answer to that problem. This has been theoretically established by some recent works (see [1]-[3]). Particularly in [3], Hutson gave quite a simple proof for this problem, with use of the asymptotic Schauder fixed point theorems. There, for the multivalued system

$$
x^{\prime} \in f(x), \quad x \in R^{n}
$$

with $f$ u.s.c. and convex-valued, he proposed the following open problem:

Does the permanence of (1.2) imply the existence of an equilibrium state?

A positive answer to this question has recently been given in [4]. However, it is of interest to enquire whether under different, or perhaps weaker conditions, the answer would still be positive. In particular for $f$ l.s.c. and not necessarily convex-valued the question still remains open, and we shall consider this in the present paper.

We first consider the functional differential inclusion

$$
x^{\prime} \in f\left(t, x_{t}\right)=f\left(t+T, x_{t}\right),
$$

Received January 31, 1994.

1991 Mathematics Subject Classification. Primary 34A60.

Key words and phrases. Functional differential inclusions, periodic solutions, permanent systems, equilibrium states. 
where $T$ is a fixed positive number, and the right-hand side is not convex. In recent years, there has been increasing interest in the theory of periodic solutions of (1.4) because of its importance in several applied areas such as mathematical economics, optimization and optimal control, and statistics, and some works can be found in [5]-[8] and the references therein. We prove here (Theorem 3.2) an existence result for periodic solutions of (1.3). We then come to (1.2) and present in section 4 a counterexample to the above conjecture when $f$ is l.s.c. and non-convex-valued. However, in section 5 we establish a somewhat weaker result, that is, the existence of a "pre-equilibrium" state for such systems.

2. Preliminaries. Let $X$ and $Y$ be metric spaces. A multifunction $G: X \rightarrow 2^{Y} \backslash \varnothing$ is said to be l.s.c. if for every $x_{0} \in X, y_{0} \in G\left(x_{0}\right)$ and $\varepsilon>0$, there exists $\delta>0$ such that

$$
G(x) \cap B\left(y_{0}, \varepsilon\right) \neq \varnothing, \quad \forall x \in B\left(x_{0}, \delta\right),
$$

where $B\left(x_{0}, \delta\right)=\left\{x \in X: \operatorname{dist}\left(x, x_{0}\right)<\delta\right\}$ and $B\left(y_{0}, \varepsilon\right)$ is similar. A map $G: X \rightarrow$ $2^{Y} \backslash \varnothing$ is said to be u.s.c. if for every $x_{0} \in X$ and every open set $U$ with $G\left(x_{0}\right) \subset U$, there exists $\delta>0$ such that

$$
G(x) \subset U, \quad \forall x \in B\left(x_{0}, \delta\right)
$$

The following results are vital to our discussion.

Theorem 2.1 (Bressan and Colombo, $[9])$. Let $(X, \mathcal{T})$ be a paracompact space, $(Y, d)$ a complete metric space and $G: X \rightarrow 2^{Y}$ a l.s.c. multifunction with nonempty closed values. Let $\mathcal{T}^{+}$be a topology on $X$ with the property:

(P) For every pair of sets $A \subset B$, with $A$ closed and $B$ open (in the original topology $\mathcal{T})$, there exists a set $C$, closed/open with respect to $\mathcal{T}^{+}$, such that $A \subset C \subset B$.

Then $G$ admits a $\mathcal{T}^{+}$-continuous selection, that is, there exists a function $g: X \rightarrow Y$ such that $g(x) \in G(x)$, for all $x \in X$ and $g(x)$ is continuous in the topology $\mathcal{T}^{+}$.

Theorem 2.2 (Horn, [10]). Let $S_{0} \subset S_{1} \subset S_{2}$ be convex subsets of a Banach space $X$ with $S_{0}$ and $S_{2}$ compact and $S_{1}$ open in $S_{2}$. Let $P: S_{2} \rightarrow X$ be a continuous map such that for some integer $N>0$,

$$
\begin{array}{ll}
P^{j}\left(S_{1}\right) \subset S_{2}, & 0 \leq j \leq N-1, \\
P^{j}\left(S_{1}\right) \subset S_{0}, & N \leq j \leq 2 N-1 .
\end{array}
$$

Then $P$ has a fixed point in $S_{0}$.

In the sequel, given a real Banach space $X$, set

$$
P_{k}(X)=\{A \subset X: \text { nonempty, compact }\} \text {. }
$$

Then in the usual Hausdorff metric $\rho, P_{k}(X)$ is a complete metric space. 
3. Periodic solutions. Consider the functional differential inclusion

$$
x^{\prime} \in f\left(t, x_{t}\right),
$$

where $x_{t}:[-r, 0] \rightarrow R^{n}$ is defined by $x_{t}(\theta)=x(t+\theta)$. We make the following assumptions.

$\left(\mathrm{H}_{1}\right) f: R \times C_{r} \rightarrow P_{k}\left(R^{n}\right)$ is l.s.c. where $C_{r}=C\left([-r, 0], R^{n}\right)$ with the usual norm $\|\cdot\|$; and $f(t+T, \cdot)=f(t, \cdot)$, for $t \in R$.

$\left(\mathrm{H}_{2}\right)$ For each $a>0$, there exists $h(a)>0$ such that

$$
\rho(f(t, \varphi), 0) \leq h(a), \quad \text { for } t \in R \text { and } \varphi \in C_{r} \text { with }\|\varphi\| \leq a .
$$

Let $I=[0, T]$. A map $x:[-r, T] \rightarrow R^{n}$ is said to be a solution of $(3.1)$ with the initial value $x_{0}=\varphi \in C_{r}$, if $x(t)$ is absolutely continuous on $I$ and satisfies (3.1) a.e. on $I$ and $x_{0}=\varphi$. If $x(t)$ is a solution of (3.1) defined on $R$ and satisfies the condition $x(t+T)=x(t)$ for $t \in R$, then $x(t)$ is called a $T$-periodic solution of (3.1).

The following theorem deals with the existence of solutions to initial-value problems.

Theorem 3.1. Let $\left(\mathrm{H}_{1}\right)$ and $\left(\mathrm{H}_{2}\right)$ be satisfied. Then for every $\varphi \in C_{r},(3.1)$ has (locally) a solution $x(t)$ with the initial value $x_{0}=\varphi$.

Proof. Without loss of generality let $\varphi \equiv 0$. Given $a>0$, by $\left(\mathrm{H}_{2}\right)$ there exists $h>0$ such that

$$
\rho(f(t, \varphi), 0) \leq h, \quad \forall(t, \varphi) \in R \times C_{r} \text { with }\|\varphi\| \leq a .
$$

Take $M>h$ and the family of all canonical neighborhoods

$$
\Gamma^{M}\left(t_{0}, \varphi_{0}, \delta\right)=\left\{(t, \psi) \in R \times C_{r}:\left\|\psi-\varphi_{0}\right\| \leq M\left(t-t_{0}\right), t_{0} \leq t<t_{0}+\delta\right\}
$$

By [9, the lemma], such neighborhoods generate a topology $\Gamma^{M}$ of $R \times C$, satisfying the property $(\mathrm{P})$, and hence by Theorem 2.1, there exists a $\Gamma^{M}$-continuous selection $\tilde{f}$ of $f$.

Set $b>0$ such that $b h<a$, and set

$$
X=\left\{y \in C\left([-r, b], R^{n}\right): y_{0}=0,\left|y\left(t_{1}\right)-y\left(t_{2}\right)\right| \leq h\left|t_{1}-t_{2}\right|, t_{1}, t_{2} \in[0, b]\right\}
$$

with the norm $\|\cdot\|_{[0 . b]}$. We claim that $\tilde{f}: X \rightarrow L^{1}\left([0, b], R^{n}\right)$ is continuous. Following the line of [11], we prove this claim. Fix $u \in X, \varepsilon>0$. By $\left(\mathrm{H}_{2}\right)$ there exists $N \geq 1$ such that

$$
\left|\tilde{f}\left(t, x_{1}\right)\right| \leq N
$$

on $\bar{B}(u, 1)$. The $\Gamma^{M}$-continuity of $\tilde{f}$ implies that for every $t \in[0, b)$, there exists $\sigma(t) \in$ $(t, b]$ such that

$$
\left|\tilde{f}\left(s, x_{t}\right)-\tilde{f}\left(t, u_{t}\right)\right| \leq \frac{\varepsilon}{4 b}
$$

whenever $t \leq s<\sigma(t),\left\|x_{s}-u_{t}\right\| \leq M(s-t)$. Using [11, the technical lemma], select finitely many disjoint intervals $I_{i}=\left[t_{i}, \sigma\left(t_{i}\right)\right), i=1, \ldots, k$ such that

$$
\operatorname{meas}\left(I_{1} \cup \cdots \cup I_{k}\right) \geq b-\frac{\varepsilon}{8 N} .
$$


Set $\delta=\varepsilon(M-h) /(8 k N)$. Then we have that $\|u-v\|_{[0 . b]} \leq \delta$ implies that

$$
\int_{0}^{b}\left|\tilde{f}\left(s, u_{s}\right)-\tilde{f}\left(s, v_{s}\right)\right| d s \leq \varepsilon \quad \text { for all } v \in X .
$$

Indeed, for every $i=1, \ldots, k$, consider the interval $J_{i}=\left[t_{i}, t_{i}+\delta /(M-h)\right)$. Set $S=\bigcup I_{i}, J=\bigcup J_{i}, S^{\mathrm{c}}=[0, b] \backslash S$. Then

$$
\operatorname{meas}(J) \leq \frac{k \delta}{M-h}, \quad \operatorname{meas}\left(S^{c}\right) \leq \frac{\varepsilon}{8 N} .
$$

Notice that if $t \in S \backslash J$, then $t \in\left[t_{i}+\delta /(M-h), \sigma\left(t_{i}\right)\right)$ for some $i$. Since $\left\|u_{t_{i}}-v_{t_{i}}\right\| \leq \delta$ and $u, v$ are Lipschitz continuous with constant $h<M$, there hold:

$$
\begin{aligned}
\left\|v_{t}-u_{t_{i}}\right\| & \leq\left\|v_{t}-v_{t_{i}}\right\|+\left\|v_{t_{i}}-u_{t_{i}}\right\| \\
& \leq \delta+\max _{\left[-r_{0} \cdot 0\right]}\left|v(t+\theta)-v\left(t_{i}+\theta\right)\right| \\
& \leq \delta+h\left(t-t_{i}\right) \leq(M-h)\left(t-t_{i}\right)+h\left(t-t_{i}\right)=M\left(t-t_{i}\right), \\
\left\|u_{t}-u_{t_{i}}\right\| & \leq h\left(t-t_{i}\right)<M\left(t-t_{i}\right) .
\end{aligned}
$$

Hence by (3.4),

$$
\begin{aligned}
\left|\tilde{f}\left(t, v_{t}\right)-\tilde{f}\left(t, u_{t}\right)\right| & \leq\left|\tilde{f}\left(t, v_{t}\right)-\tilde{f}\left(t_{i}, u_{t_{i}}\right)\right|+\left|\tilde{f}\left(t_{i}, u_{t_{i}}\right)-\tilde{f}\left(t, u_{t}\right)\right| \\
& \leq \frac{\varepsilon}{2 b}
\end{aligned}
$$

From (3.3), (3.5), and (3.6) it follows that

$$
\begin{aligned}
\int_{0}^{b}\left|\tilde{f}\left(s, u_{s}\right)-\tilde{f}\left(s, v_{s}\right)\right| d s & \leq \int_{S \backslash J}\left|\tilde{f}\left(s, u_{s}\right)-\tilde{f}\left(s, v_{s}\right)\right| d s+\int_{S^{\prime} \cup J}\left|\tilde{f}\left(s, u_{s}\right)-\tilde{f}\left(s, v_{s}\right)\right| d s \\
& \leq b \cdot \frac{\varepsilon}{2 b}+2 N\left[\operatorname{meas}(J)+\operatorname{meas}\left(S^{c}\right)\right] \leq \varepsilon
\end{aligned}
$$

which proves the continuity of $\tilde{f}$ on $X$. The existence of the solution can be obtained by applying Schauder's fixed point theorem to $X$, for the equation $x^{\prime}=\tilde{f}\left(t, x_{t}\right)$. This completes the proof.

The following is the main result of this section, which generalizes a well-known result of Yoshizawa (see [12] and [13]) on the existence of periodic solutions for functional differential equations to functional differential inclusions with nonconvex right-hand sides.

Let $D_{1}, D_{2} \subset R^{n}$. Solutions of (3.1) are said to be bounded in $D_{1}$ if there exists $M>0$ such that every solution $x(t)$ of $(3.1)$ with the initial value $x_{0}=\varphi$ satisfies

$$
|x(t)| \leq M, \quad \forall t \geq 0,
$$

whenever $\varphi \in C_{r}$ and $\varphi(\theta) \in D_{1}, \theta \in[-r, 0]$. Solutions of (3.1) are said to be locally dissipative relative to $D_{1}-D_{2}$, if for every $\varphi \in C_{r}$ with $\varphi(\theta) \in D_{1}, \theta \in[-r, 0]$, there exist $\delta_{\varphi}, t_{\varphi} \geq 0$ such that every solution of (3.1) with the initial value $x_{0}=\psi$ satisfies

$$
x(t) \in D_{2}, \quad \forall t \geq t_{\varphi}, \psi \in B\left(\varphi, \delta_{\varphi}\right)
$$

and

$$
\psi(\theta) \in D_{1}, \quad \theta \in[-r, 0]
$$


Theorem 3.2. Let $\left(\mathrm{H}_{1}\right)$ and $\left(\mathrm{H}_{2}\right)$ be satisfied. Let $D_{1}, D_{2} \subset R^{n}, D_{1}$ be bounded, convex and open, $D_{2} \subset D_{1}$ be closed. If solutions of (3.1) are bounded in $D_{1}$ and locally dissipative relative to $\bar{D}_{1}-D_{2}$, then (3.1) has a $T$-periodic solution lying in $D_{2}$.

Proof. Since solutions of $(3.1)$ are bounded in $D_{1}$, there exists $M_{1}>0$ such that every solution $x(t)$ of $(3.1)$ with $x_{0}=\varphi$ lying in $D_{1}$ satisfies

$$
|x(t)| \leq M_{1}, \quad \forall t \geq 0 .
$$

Hence there exists $h \geq 1$ such that

$$
\rho(f(t, \varphi), 0) \leq h, \quad \text { for } \varphi \in C_{r} \text { with }\|\varphi\| \leq M_{2},
$$

where $M_{2}=M_{1}+\sup _{D_{1}}|y|+1$. Set $X=C\left([-r, T], R^{n}\right)$. By Theorem 2.1, for $M>$ $M_{1}+h+\sup _{D_{1}}|y|$, there exists a $\Gamma^{\Lambda I}$-continuous selection $\tilde{f}(t, \varphi)$ of $f(t, \varphi)$ on $[0, T] \times C_{r}$. Define $f_{1}: R \times C_{r} \rightarrow R^{n}$ by

$$
f_{1}(t, \varphi)= \begin{cases}\tilde{f}(t, \varphi), & t \in[0, T], \\ \tilde{f}(t-k T, \varphi), & t \in[k T,(k+1) T), k= \pm 1, \pm 2, \ldots\end{cases}
$$

Obviously, for solutions of $x^{\prime}=f_{1}\left(t, x_{t}\right),(3.7)$ still holds.

Set

$$
\begin{aligned}
S= & \left\{\varphi \in C_{r}:\|\varphi\| \leq M_{2},\left|\varphi\left(\theta_{1}\right)-\varphi\left(\theta_{2}\right)\right| \leq(h+1)\left|\theta_{1}-\theta_{2}\right|, \theta_{1}, \theta_{2} \in[-r, 0]\right\}, \\
V=\left\{x \in X: x_{0} \in S,\left|x\left(t_{1}\right)-x\left(t_{2}\right)\right| \leq(h+1)\left|t_{1}-t_{2}\right|,\right. & \\
& \left.t_{1}, t_{2} \in[0, T],\|x\|_{[0 . T]}=\max _{[0, T]}|x(t)| \leq M_{2}\right\} .
\end{aligned}
$$

Then by the proof of Theorem 3.1, $f_{1}:[0, T] \times V \rightarrow L^{1}\left([0, T], R^{n}\right)$ is continuous. According to the Arzela-Ascoli theorem, $S$ is compact. Using the finite covering theorem and local dissipativeness, we get that there exists a positive integer $N>T+r+1$ such that every solution $x(t)$ of

$$
x^{\prime}=f_{1}\left(t, x_{t}\right), \quad x_{0}=\varphi
$$

satisfies

$$
x(t) \in D_{2}, \quad \forall t \geq N T,
$$

whenever $\varphi \in S_{2}$ with $\varphi(\theta) \in \bar{D}_{1}, \theta \in[-r, 0]$.

Take any partition

$$
t_{0}=0>t_{1}>\cdots>t_{m}=-r, \quad t_{i}-t_{i+1}=\frac{r}{m}=\Delta_{m}, i=0, \ldots, m-1, m>1 .
$$

For each $\varphi \in C_{r}$, define

$$
\bar{\varphi}_{m}(\theta)= \begin{cases}\varphi(0)-\frac{\theta}{\Delta_{m}}\left[\varphi\left(t_{1}\right)-\varphi\left(t_{0}\right)\right], & \theta \in\left[t_{1}, t_{0}\right], \\ \cdots & \theta \in\left[t_{i+1}, t_{i}\right], \\ \varphi\left(t_{i}\right)-\frac{\theta-t_{i}}{\Delta_{m}}\left[\varphi\left(t_{i+1}\right)-\varphi\left(t_{i}\right)\right], & \\ \cdots & \theta \in\left[t_{m}, t_{m-1}\right],\end{cases}
$$




$$
K(\varphi)=\left(\varphi\left(t_{m}\right), \varphi\left(t_{m-1}\right), \ldots, \varphi\left(t_{0}\right)\right) \in R^{(m+1) n} .
$$

Obviously, $K: C_{r} \rightarrow R^{(m+1) n}$ is completely continuous. Set

$$
f_{1 m}(t, K(\varphi))=f_{1}\left(t, \bar{\varphi}_{m}\right), \quad \text { for } \varphi \in C_{r}
$$

From the construction of $f_{1 m}$ it is easily seen that $f_{1 m}:[0, T] \times V \rightarrow L^{1}\left([0, T], R^{n}\right)$ is continuous. Notice that for each $\varphi \in S$, there exists $0 \leq i_{m} \leq m-1$ such that

$$
\begin{aligned}
\left\|\varphi-\bar{\varphi}_{m}\right\| & =\max _{\left[t_{i_{m}+1 . t_{m}}\right]}\left|\varphi(\theta)-\varphi\left(t_{i_{m}}\right)-\frac{\theta-t_{i_{m}}}{\Delta_{m}}\left[\varphi\left(t_{i_{m}+1}\right)-\varphi\left(t_{i_{m}}\right)\right]\right| \\
& \leq 2(h+1) \Delta_{m} .
\end{aligned}
$$

Hence

$$
\left\|\varphi-\bar{\varphi}_{m}\right\| \rightarrow 0, \quad \text { as } m \rightarrow \infty \text { uniformly with respect to } \varphi \in S .
$$

It follows that

$$
\int_{0}^{T}\left|f_{1}\left(s, x_{s}\right)-f_{1 m}\left(s, K\left(x_{s}\right)\right)\right| d s \rightarrow 0 \quad \text { as } m \rightarrow \infty, \text { uniformly in } x \in V .
$$

Since the equation $x^{\prime}=f_{1 m}\left(t, K\left(x_{t}\right)\right)$ is one with several delays, by the Arzela-Ascoli theorem, [14, Proposition 4.2], (3.7), (3.8), and (3.10), we have that for sufficiently large $m$, say $m \geq 1$, every solution $x(t)$ of the Cauchy problem

$$
x^{\prime}=f_{1 m}\left(t, K\left(x_{t}\right)\right), \quad x_{0}=\varphi
$$

satisfies

$$
\begin{array}{ll}
|x(t)| \leq M_{1}+\frac{1}{4} & \text { for } t \in[0, N T], \\
\operatorname{dist}\left(x(t), D_{2}\right) \leq \frac{\varepsilon_{0}}{4} & \text { for } t \in\left[N T, 2 N_{1} T\right],
\end{array}
$$

whenever $\varphi \in S$ and $\varphi(\theta) \in \bar{D}_{1}, \theta \in[-r, 0]$. Here $\varepsilon_{0}=\operatorname{dist}\left(\partial D_{1}, D_{2}\right)$, and $N_{1}$ is an integer with $N_{1}>(N+1)(T+r+1)$.

Consider a nonnegative $C^{x}$ function $\psi: R^{(m+1) n+1} \rightarrow R^{+}$satisfying

$$
\begin{gathered}
\operatorname{supp}(\psi) \subset\left\{(t, y): t \in[-1,0], y \in R^{(m+1) n} \text { with }|y| \leq-M t\right\}, \\
\iint_{R^{(m+1) n+1}} \psi(t, y) d y d t=1 .
\end{gathered}
$$

Set $Q=[0, T] \times R^{(m+1) n}$. For every $\tau \geq 1$, set $\psi_{\tau}(t, y)=\tau^{(m+1) n+1} \psi(\tau t, \tau y)$ and define the convolution

$$
F_{m \tau}(t, y)=f_{1 m} * \psi_{\tau}(t, y)=\iint_{Q} f_{1 m}(s, z) \psi_{\tau}(t-s, y-z) d s d z
$$

$F_{m \tau}$ is obviously of class $C^{\infty}$. By [14, Proposition 4.2],

$$
\begin{array}{r}
\int_{0}^{T}\left|F_{m \tau}\left(s, u\left(s-t_{m}\right), \ldots, u\left(s-t_{0}\right)\right)-f_{1 m}\left(s, u\left(s-t_{m}\right), \ldots, u\left(s-t_{0}\right)\right)\right| d s \rightarrow 0 \\
\tau \rightarrow \infty, \text { uniformly in } u \in V .
\end{array}
$$


Define

$$
\widetilde{F}_{m \tau(t, y)}= \begin{cases}F_{m \tau}(t, y), & t \in[0, T], \\ F_{m \tau}(t-k T, y), & t \in[k T,(k+1) T], \quad k= \pm 1, \pm 2, \ldots\end{cases}
$$

From (3.12) and (3.13) it follows that for every $\varphi \in S$ with $\varphi(\theta) \in \bar{D}_{1}, \theta \in[-r, 0]$, the unique solution $x(t, \varphi)$ of the Cauchy problem

$$
x^{\prime}(t)=\widetilde{F}_{m \tau}\left(t, x\left(t-t_{m}\right), \ldots, x\left(t-t_{0}\right)\right) \equiv \widetilde{F}_{m \tau}[x](t), \quad x_{0}=\varphi
$$

satisfies

$$
\begin{array}{ll}
|x(t, \varphi)| \leq M_{1}+\frac{1}{2}, & \text { for } t \in[0, N T], \\
\operatorname{dist}\left(x(t, \varphi), D_{2}\right) \leq \frac{\varepsilon_{0}}{2}, & \text { for } t \in\left[N T, 2 N_{1} T\right],
\end{array}
$$

whenever $\tau$ is sufficiently large, say $\tau \geq 1$.

Set

$$
\begin{aligned}
S_{0}= & \left\{\varphi \in S: \varphi(\theta) \in \bar{B}\left(D_{2}, \frac{\varepsilon_{0}}{2}\right),\right. \\
& \left.\left|\varphi\left(\theta_{1}\right)-\varphi\left(\theta_{2}\right)\right| \leq\left(h+\frac{1}{4}\right)\left|\theta_{1}-\theta_{2}\right|, \theta, \theta_{1}, \theta_{2} \in[-r, 0]\right\}, \\
S_{1}= & \left\{\varphi \in S: \varphi(\theta) \in D_{1},\right. \\
& \left.\left|\varphi\left(\theta_{1}\right)-\varphi\left(\theta_{2}\right)\right|<\left(h+\frac{1}{2}\right)\left|\theta_{1}-\theta_{2}\right|, \theta, \theta_{1}, \theta_{2} \in[-r, 0]\right\} .
\end{aligned}
$$

Clearly, $S_{0}$ is compact convex, $S_{1}$ is open convex, and $S_{0} \subset S_{1} \subset S$. Define a Poincaré map $P: S \rightarrow C_{r}$, by

$$
P(\varphi)(\theta)=x(T+\theta, \varphi), \quad \theta \in[-r, 0] .
$$

From (3.15) it follows that

$$
P^{j}\left(S_{1}\right) \subset S, \quad \text { for } 0 \leq j \leq N_{1}-1
$$

and

$$
P^{j}\left(S_{1}\right) \subset S_{0}, \quad \text { for } N_{1} \leq j \leq 2 N_{1}-1 .
$$

Then by Horn's fixed point theorem (Theorem 2.2), $P$ has a fixed point $\varphi_{m \tau} \in S_{0}$. The uniqueness of the solution with initial value implies

$$
x\left(t+T, \varphi_{m \tau}\right)=x\left(t, \varphi_{m \tau}\right),
$$

which shows that $x\left(t, \varphi_{m \tau}\right)$ is a $T$-periodic solution of (3.14). Applying (3.13) and the Arzela-Ascoli theorem to the sequence $\left\{\varphi_{m \tau}\right\}$, passing to a subsequence if necessary, we may assume

$$
\varphi_{m \tau} \rightarrow \varphi_{m}^{*} \quad \text { in } S_{0}
$$


and

$$
x\left(t, \varphi_{m \tau}\right) \rightarrow x_{m}(t) \text { as } \tau \rightarrow \infty \text {, uniformly in } R,
$$

where $x_{m}(t)$ is a solution of $x^{\prime}=f_{1 m}\left(t, K\left(x_{t}\right)\right)$ with the initial value $x_{0}=\varphi_{m}^{*}$ and the period $T$. Indeed, using (3.13) yields

$$
\begin{aligned}
x\left(t, \varphi_{m \tau}\right)= & x\left(0, \varphi_{m \tau}\right)+\int_{0}^{t} \widetilde{F}_{m \tau}\left[x\left(\cdot, \varphi_{m \tau}\right)\right](s) d s \\
& \rightarrow x_{m}(0)+\int_{0}^{t} f_{1 m}\left(s, K\left(\left(x_{m}\right)_{s}\right)\right) d s=x_{m}(t), \tau \rightarrow \infty \text { uniformly on }[0, T]
\end{aligned}
$$

Similarly, by (3.10), we may assume

$$
\varphi_{m}^{*} \rightarrow \varphi^{*} \text { in } S_{0}, \quad x_{m}(t) \rightarrow x^{*}(t), m \rightarrow \infty, \text { uniformly in } R,
$$

where $x^{*}(t)$ is a $T$-periodic solution of $x^{\prime}=f_{1}\left(t, x_{t}\right)$, and hence of $(3.1)$, with $x_{0}=\varphi^{*}$. This completes the proof.

4. A counterexample. The following counterexample provides a negative answer to Hutson's open problem, for multivalued permanent systems with nonconvex right-hand sides.

Consider the inclusion (see Fig. 1)

$$
x^{\prime} \in f(x), \quad x \in R
$$

where

$$
f(x)= \begin{cases}\{1\} & (x \leq 1) \\ \{-1,1\} & (1<x<2) \\ \{-1\} & (x \geq 2) .\end{cases}
$$

It is easy to prove that $f: R \rightarrow P_{k}(R)$ is l.s.c. The solution curves of $(4.1)$ can be described as in Fig. 2.

Since for every $x_{0}>2$, the solution $x(t)$ of (4.1) with $x(0)=x_{0}$ is: $x(t)=x_{0}-t$; while for every $x_{0}<1$, the solution $x(t)$ of $(4.1)$ with $x(0)=x_{0}$ is: $x(t)=x_{0}+t$. Therefore, the solutions of $(4.1)$ are $[0,3]-[1,2]$ dissipative, hence permanent (see section 5). But there is no equilibrium state of $(4.1)$ in $[1,2]$.

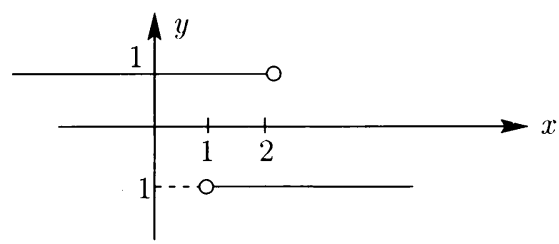

FIG. 1. $y=f(x)$ 


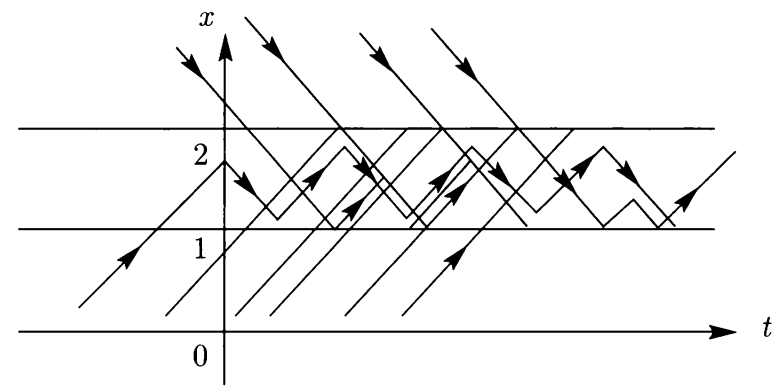

FIG. 2. Solution curves of (4.1)

5. Pre-equilibrium states. Consider the multivalued system of the type

$$
x^{\prime} \in F\left(x_{t}\right),
$$

where $F: C_{r} \rightarrow P_{k}\left(R^{n}\right)$ is l.s.c. We also need the following assumption.

$\left(\mathrm{H}_{3}\right)$ For each $a>0$, there exists $h(a)>0$ such that

$$
\rho(F(\varphi), 0) \leq h(a), \quad \forall \varphi \in C_{r}, \text { with }\|\varphi\| \leq a .
$$

The system (5.1) is said to be permanent, if there exist constants $m_{1}>m_{2}>0$ such that solutions are locally dissipative relative to $R_{+}^{n}-\left[m_{2}, m_{1}\right]^{n}$, where $R_{+}^{n}=\left\{x \in R^{n}\right.$ : $\left.x_{i} \geq 0, i=1, \ldots, n\right\}$ and $\left[m_{2}, m_{1}\right]^{n}=\left\{x \in R^{n}: m_{2} \leq x_{i} \leq m_{1}, i=1, \ldots, n\right\}$.

The main result of this section is the following

ThEOREM 5.1. Assume (5.1) is permanent and its solutions are uniformly bounded. Then (5.1) has a pre-equilibrium $x^{*} \in\left[m_{2}, m_{1}\right]^{n}$, for some constants $m_{1}>m_{2}>0$, that is,

$$
0 \in \overline{\mathrm{CO}} F\left(x^{*}\right) .
$$

Proof. The permanence of (5.1) implies that there exist $m_{1}>m_{2}>0$ such that solutions of (5.1) are locally dissipative relative to $R_{+}^{n}-\left[m_{2}, m_{1}\right]^{n}$. Set

$$
\begin{aligned}
& D_{1}=\left\{x \in R^{n}: \frac{m_{2}}{2}<x_{i}<m_{1}+1, i=1, \ldots, n\right\}, \\
& D_{2}=\left\{x \in R^{n}: m_{2} \leq x_{i} \leq m_{1}, i=1, \ldots, n\right\} .
\end{aligned}
$$

Continue to use the notation and the arguments in the proof of Theorem 3.2. By uniform boundedness, there is $M_{1}>0$ such that (3.7) holds for the solutions of (4.1). By $\left(\mathrm{H}_{3}\right)$, there exists $h \geq 1$ such that

$$
\rho(F(\varphi), 0) \leq h, \quad \forall \varphi \in C_{r} \text { with }\|\varphi\| \leq M_{2}
$$

where $M_{2}=M_{1}+m_{1}+2$. Fix any $T \in(0,1]$. Set $U_{T}=V$. Applying Theorem 2.1, we see that for $M>M_{1}+h+m_{1}+2$, there exists a $\Gamma^{M}$-continuous selection $F_{1 T}$ of $F$ on $C_{r}$. Then $F_{1 T}: U_{T} \rightarrow L^{1}\left([0, T], R^{n}\right)$ is continuous. Hence there exists a positive integer $N>r+2$ such that every solution $x(t)$ of

$$
x^{\prime}=F_{1 T}\left(x_{t}\right), \quad x_{0}=\varphi
$$


satisfies

$$
x(t) \in D_{2}, \quad \forall t \geq N
$$

for $\varphi \in S$ with $\varphi(\theta) \in \bar{D}_{1}, \theta \in[-r, 0]$. Choose a $C^{\infty}$ function $\psi: R^{(m+1) n} \rightarrow R^{+}$ satisfying

$$
\begin{gathered}
\operatorname{supp}(\psi) \subset\left\{y \in R^{(m+1) n}:|y| \leq-M t, t \in[-1,0]\right\}, \\
\iint_{R^{(m+1) n}} \psi(y) d y=1 .
\end{gathered}
$$

For every $\tau \geq 1$, set $\psi_{\tau}(y)=\tau^{(m+1) n} \psi(\tau y)$ and define

$$
F_{T m \tau}(y)=F_{1 T m} * \psi_{\tau}(y)=\iint_{R^{(m+1) n}} F_{1 T m}(z) \psi_{\tau}(y-z) d z,
$$

where $F_{1 T m}(K(\varphi))=F_{1 T}\left(\bar{\varphi}_{m}\right)$, for $\varphi \in C_{r}$. Then $F_{T m \tau}$ is of class $C^{x}$.

Consider the differential-difference equation

$$
x^{\prime}(t)=F_{T m \tau}\left(x\left(t-t_{m}\right), \ldots, x\left(t-t_{0}\right)\right) .
$$

Set $\varepsilon_{k}=\frac{1}{2 k} \min \left(\frac{m_{2}}{2}, 1\right), k=2,3, \ldots$ Similar to the proof of Theorem 3.2, we have that for every $k$, there exists a positive integer $M(k)$ such that the solution $x(t, \varphi)$ of $(5.3)$ with the initial value $x_{0}=\varphi$ satisfies

$$
\begin{gathered}
|x(t, \varphi)| \leq M_{1}+\varepsilon_{k}, \quad \text { for } t \in[0, N], \\
\operatorname{dist}\left(x(t, \varphi), D_{2}\right) \leq \varepsilon_{k}, \quad \text { for } t \in[N, 2(N+r+2)],
\end{gathered}
$$

whenever $\varphi \in S$ with $\varphi(\theta) \in \bar{D}_{1}, \theta \in[-r, 0]$, and $m, \tau \geq M(k)$. Set

$$
\begin{aligned}
S_{0}= & \left\{\varphi \in S: \operatorname{dist}\left(\varphi(\theta), D_{2}\right) \leq \varepsilon_{k},\right. \\
& \left.\left|\varphi\left(\theta_{1}\right)-\varphi\left(\theta_{2}\right)\right| \leq\left(h+\frac{1}{4}\right)\left|\theta_{1}-\theta_{2}\right|, \theta, \theta_{1}, \theta_{2} \in[-r, 0]\right\}, \\
S_{1}= & \left\{\varphi \in S: \varphi(\theta) \in D_{1},\right. \\
& \left.\left|\varphi\left(\theta_{1}\right)-\varphi\left(\theta_{2}\right)\right|<\left(h+\frac{1}{2}\right)\left|\theta_{1}-\theta_{2}\right|, \theta, \theta_{1}, \theta_{2} \in[-r, 0]\right\} .
\end{aligned}
$$

For each $m, \tau \geq M(k)$ define the Poincaré map $P_{m \tau}$ by

$$
P_{m \tau}(\varphi)(\theta)=x(T+\theta, \varphi), \quad \theta \in[-r, 0] .
$$

Take a positive integer $N(T)$ such that $N(T) T \in[N+r, N+r+1]$. Then using (5.4), we get

$$
P_{m \tau}^{j}\left(S_{1}\right) \subset S, \quad \text { for } 0 \leq j \leq N(T)-1,
$$


and

$$
P_{m \tau}^{j}\left(S_{1}\right) \subset S_{0}, \quad \text { for } N(T) \leq j \leq 2 N(T)-1 .
$$

By Horn's fixed point theorem, $P_{m \tau}$ has a fixed point $\varphi_{T m \tau} \in S_{0}$. That is, $x\left(t, \varphi_{T m \tau}\right)$ is a $T$-periodic solution of (5.3), for $m$ and $\tau$. Notice

$$
x\left(t+\theta, \varphi_{m \tau T}\right) \in S_{0}, \quad t \in R \quad \text { and } \quad \theta \in[-r, 0] .
$$

Applying the Arzela-Ascoli theorem, we know

$$
{\overline{\left\{\varphi_{T m \tau}\right\}_{T \in(0,1]}}} \subset S_{0} .
$$

Using the Arzela-Ascoli theorem and [14, Proposition 4.2], passing to a subsequence if necessary, we may assume that

$$
\varphi_{T m \tau} \rightarrow \varphi_{T} \quad \text { in } S_{0}
$$

and

$$
x\left(t, \varphi_{T m \tau}\right) \rightarrow x\left(t, \varphi_{T}\right), m, \tau \rightarrow \infty \text {, uniformly in } R,
$$

where $x\left(t, \varphi_{T}\right)$ is a $T$-periodic solution of $x^{\prime}=F_{1 T}\left(x_{t}\right)$ lying in $S_{0}$. Set $\left\{T_{j}\right\} \subset(0,1]$ such that $T_{j} \rightarrow 0, j \rightarrow \infty$. Similarly, we may assume that $\varphi_{T_{j}} \rightarrow \varphi$ in $C_{r}$ and $x\left(t, \varphi_{T_{j}}\right) \rightarrow x(t)$, $j \rightarrow \infty$, uniformly on $R$. Since $\left\{F_{1 T_{j}}\left(x_{t}\left(\cdot, \varphi_{T_{j}}\right)\right)\right\}_{j}$ is bounded, passing to a subsequence if necessary we may assume that for some bounded and measurable $F_{0}(t)$ on $[0,1], F_{j}(t) \equiv$ $F_{1 T_{j}}\left(x_{t}\left(\cdot, \varphi_{T_{j}}\right)\right)$ converges weakly to $F_{0}(t)$ on $[0,1]$, and $F_{0} \in \overline{\mathrm{CO}} F_{j}$. Notice

$$
\begin{aligned}
& T_{j} \rightarrow 0, \quad x\left(t+T_{j}, \varphi_{r_{j}}\right) \equiv x\left(t, \varphi_{T_{j}}\right) \\
& \left|F_{j}(t)\right| \leq h, \\
& x\left(t, \varphi_{T_{j}}\right)=x\left(0, \varphi_{T_{j}}\right)+\int_{0}^{t} F_{j}(s) d s \text { for } t \in[0,1] .
\end{aligned}
$$

Therefore, $x(t) \equiv x^{*} \in R^{n} \cap S_{0}$. Hence from

$$
x(t)=x(0)+\int_{0}^{t} F_{0}(s) d s
$$

it follows that $F_{0}(t)=0$, a.e. $[0,1]$. This completes the proof.

Acknowledgment. The authors thank Professor Ding Tongren for providing a counterexample stated in section 4 . They also thank the referee for helpful suggestions.

\section{REFERENCES}

[1] J. Hofbauer and K. Sigmund, Dynamical Systems and the Theory of Evolution, Cambridge University Press, 1988

[2] V. Hutson and W. Moran, Persistence of species obeying difference equations, Math. Biosci. 15, 203-213 (1982)

[3] V. Hutson, The existence of an equilibrium for permanent systems, Rocky Mountain J. Math. 20, 1033-1040 (1990) 
[4] Li Yong, Wang Huaizhong, and Lu Xianrui, Equilibrium of permanent multivalued systems, Quart. Appl. Math. 51, 791-795 (1993)

[5] G. Haddad and M. Lasry, Periodic solutions of functional differential inclusions and fixed points of $\sigma$-selectionable correspondences, J. Math. Anal. Appl. 96, 295-312 (1983)

[6] Jack W. Macki, P. Nistri, and P. Zecca, The existence of periodic solutions to nonautonomous differential inclusions, Proc. Amer. Math. Soc. 104, 840-844 (1988)

[7] S. Plaskacz, Periodic solutions of nonlinear functional differential inclusions on compact subsets of $R^{n}$, J. Math. Anal. Appl. 148, 202-212 (1990)

[8] M. Frigon and A. Granas, Existence theorems for differential inclusions without convexity, C. R. Acad. Sci. Paris Ser. I. Math. 310, 819822 (1990) (in French)

[9] A. Bressan and G. Colombo, Selections and representations of multifunctions in paracompact spaces, Studia Math. 102, 209-216 (1992)

[10] W. A. Horn, Some fixed point theorem for compact maps and flows in Banach spaces, Trans. Amer. Math. Soc. 149, 391-404 (1970)

[11] A. Bressan, Directionally continuous selections and differential inclusions, Funkcial. Ekvac. 31, 459-470 (1988)

[12] T. Yoshizawa, Stability Theory by Liapunov's Second Method, Math. Soc. Japan, Tokyo, 1966

[13] T. A. Burton and S. Zhang, Unified boundedness, periodicity, and stability in ordinary and functional differential equations, Ann. Math. Pure Appl. CXLV, 129-258 (1986)

[14] A. Bressan, On the qualitative theory of lower semicontinuous differential inclusions, J. Differential Equations 77, 379-391 (1989) 